\title{
BEST APPROXIMATION IN ORLICZ SPACES
}

\author{
H. AL-MINAWI and S. AYESH \\ Department of Mathematics \\ Kuwalt University \\ P.0. BoX 5969,130 Safat \\ Kuwalt
}

(Received April 17, 1989 and in revised form December 23, 1989)

ABSTRACT. Let $X$ be a real Banach space and $(\Omega, \mu)$ be a finite measure space and $\phi$ be a strictly increasing convex continuous function on $[0, \infty)$ with $\phi(0)=0$. The space $L_{\phi}(\mu, X)$ is the set of all measurable functions $f$ with values in $X$ such that $\int_{\Omega} \phi\left(\left\|c^{-1} f(t)\right\|\right) d \mu(t)<\infty$ for some $c>0$. One of the main results of this paper is:

"For a closed subspace $Y$ of $X, L_{\phi}(\mu, Y)$ is proximinal in $L_{\phi}(\mu, X)$ if and only if $\mathrm{L}^{1}(\mu, \mathrm{Y})$ is proximinal in $\mathrm{L}^{1}(\mu, \mathrm{X})^{\prime \prime}$. As a result if $\mathrm{Y}^{\phi}$ is reflexive subspace of $\mathrm{X}$, then $\mathrm{L}_{\phi}(\phi, Y)$ is proximinal in $\mathrm{L}_{\phi}(\mu, \mathrm{X})$. Other results on proximinality of subspaces of $L_{\phi}(\mu, X)$ are proved.

1. INTRODUCTION.

Let $\phi$ be a convex orlicz function, $1 . e . \phi$ is a continuous, strictly increasing convex function defined on $[0, \infty)$ with $\phi(0)=0$ and 1 et $(\Omega, \mu)$ be a finite measure. For a real Banach space $X$, let

$$
L_{\phi}(\mu, X)=\text { measurable function } f: \Omega \rightarrow X: \int_{\Omega} \phi\left(\left\|c^{-1} f(t)\right\|\right) d \mu(t)<\infty
$$

for some $c>0$. Define a norm on $\mathrm{L}_{\phi}(\mu, \mathrm{X})$ by

$$
\|f\|_{\phi}=\operatorname{lnf}\left\{c>0: \int_{\Omega} \phi\left(\left\|c^{-1} f(t)\right\|\right) d \mu(t)<1\right\}
$$

A subspace $Y$ in a Banach space $X$ is called proximinal if for each $x \in X$ there is at least one $y \varepsilon Y$ such that $\|x-y\|=d(x, y)=1$ nf $\{\|x-h\|, h \varepsilon Y\}$. The element $y$ is called best approximant of $X$ in $Y$. Set $P(X, Y)=\{y \in Y: d(x, y)=\|x-y\|\}$.

In this paper we prove that for a closed subspace $Y$ of a Banach space $X$, $\mathrm{L}_{\phi}(\mu, \mathrm{Y})$ is proximinal in $\mathrm{L}_{\phi}(\mu, \mathrm{X})$ if and only if $\mathrm{L}^{1}(\mu, \mathrm{Y})$ is proximinal in $L^{1}(\mu, X)$. In [1] Deeb and Khali1, have shown the same result for the linear metric space $L_{\phi}(\mu, X)$ with $\phi$ modulus function and some Banach space $X$. As a consequence, if $Y$ 
is a reflexive subspace of a Banach space $X$ then $L_{\phi}(\mu, Y)$ is proximinal in $L_{\phi}(\mu, X)$.

The proximinality of some closed subspaces in $X$ are discussed. Throughout this paper $\Omega$ will be the unit interval $[0,1], \phi$ convex, strictly increasing with $\phi(0)=0, \phi(1)=1$ and $x$ is a Banach space. See Deeb and Khali1 $[1,2,3]$, Light and Cheney [4], and Khalil [5] for more detalls about proximinaltey and related topics.

2. PROXIMINALITY IN $\mathrm{L}_{\phi}(\mu, \mathrm{X})$.

LEMMA 2.1. If $\phi$ is convex, then $L_{\phi}(\mu, X) \subseteq L^{1}(\mu, X)$.

PROOF. Let $f \in L_{\phi}(\mu, X)$, then

$\int_{0}^{1} \phi\left(\left\|c^{-1} f(t)\right\|\right) d \mu(t)<M$ for some $c$ and some $M$

By Jensen's Inequality, [6]

$$
\phi\left(\int_{0}^{1}\left\|c^{-1} f(t)\right\| d \mu(t)\right)<\int_{0}^{1} \phi\left(\left\|c^{-1} f(t)\right\|\right) d \mu(t)<M
$$

or

$$
\phi\left(\int_{0}^{1}\left\|c^{-1} f(t)\right\| d \mu(t)\right)<M .
$$

Hence

$$
\int_{0}^{1}\left\|c^{-1} f(t)\right\| d \mu(t)<\phi^{-1}(M) .
$$

Therefore

$$
\int_{0}^{1}\|f(t)\| d \mu(t)<c \phi^{-1}(M)<\infty .
$$

Hence $\mathrm{f} \varepsilon \mathrm{L}^{1}(\mu, \mathrm{X})$.

LEMMA 2.2. Let $Y$ be a subspace of $X$, then for each $f \in L_{\phi}(\mu, X)$

$$
\operatorname{dist}\left(f, L_{\phi}(\mu, Y)\right)=\operatorname{lnf}\left\{c>0: \int_{0}^{1} \phi\left|c^{-1} \operatorname{dist}(f(t), Y)\right| d \mu(t)<1\right\} \text {. }
$$

PROOF. For any $g \in L_{\phi}(\mu, Y)$ we have,

$$
\begin{aligned}
\|f-g\|_{\phi} & =\operatorname{lnf}\left\{c>0: \int_{0}^{1}\left(\left\|c^{-1}(f(t)-g(t))\right\|\right) d \mu(t)<1\right\} \\
& >\operatorname{lnf}\left\{c>0: \int_{0}^{1} \phi\left(\left|c^{-1} \operatorname{dist}(f(t), Y)\right|\right) d \mu(t)<1\right\} .
\end{aligned}
$$

By taking the inf imum over $g \in L_{\phi}(\mu, Y)$ we get

$$
\operatorname{dist}\left(f, L_{\phi}(\mu, Y)\right)>\inf \left\{c>0: \int_{0}^{1} \phi\left(\left|c^{-1} \operatorname{dist}(f(t), Y)\right|\right) \operatorname{d} \phi(t)<1\right\} .
$$


Conversely, let $\varepsilon>0$ and let $f^{\prime}$ be a simple function in $L_{\phi}(\mu, x)$, such that $\left\|\mathrm{f}^{-\mathrm{f}^{\prime}}\right\|_{\phi}<\varepsilon . \quad$ Write $\mathrm{f}^{\prime}=\sum_{i=1}^{n} x_{1} \mathrm{x}_{i}$, where $\mathrm{x}_{i} \varepsilon \mathrm{x}$ and $x_{i}$ are the characteristic functions on $A_{i}$ which are disjoint measurable sets in $[0,1]$. It is clear that $f^{\prime} \varepsilon L_{\phi}(\mu, X)$. Select $h_{1} \varepsilon Y$ such that

$$
\phi\left(c^{-1}\left\|x_{i}-h_{i}\right\|\right)<\phi\left[c^{-1} \operatorname{dist}\left(x_{i}, Y\right)+\varepsilon\right], \text { for some } c>0 \text {. }
$$

Let $g=\sum_{i=1}^{n} x_{1} h_{1}$, then

$$
\int_{0}^{1} \phi\left(\left\|c^{-1} g(t)\right\|\right) \mathrm{d} \mu(t)=\sum_{i=1}^{n} \phi\left(\left\|c^{-1} h_{i}\right\|\right) \mu\left(A_{i}\right)<\infty .
$$

Hence $g \in \mathrm{L}_{\phi}(\mu, \mathrm{Y})$, then

$$
\|f-g\|_{\phi}=\left\|f-f^{\prime}+f^{\prime}-g\right\|_{\phi} \leqslant \varepsilon+\left\|f^{\prime}-g\right\|_{\phi}
$$

But $\operatorname{dist}\left(f, L_{\phi}(\mu, Y)\right)<|| f-g \|_{\phi}$

$$
\begin{aligned}
& <\varepsilon+\inf \left\{c>0: \int_{0}^{1} \phi\left(c^{-1}|| f^{\prime}(t)-g(t) \|\right) d \mu(t)<1\right\} \\
& =\varepsilon+\operatorname{Inf}\left\{c>0: \sum_{i=1}^{n} \int_{0}^{1} \phi\left(c^{-1}\left\|\mid x_{1}-h_{i}\right\|\right) d \mu(t)<1\right\} \\
& =\phi+\operatorname{lnf}\left\{c>0: \sum_{i=1}^{n} \phi\left(c^{-1}|| x_{i}-h_{i} \|\right) \mu\left(A_{i}\right)<1\right\} \\
& \left\langle\varepsilon+\inf \left\{c>0: \sum_{1=1}^{n} \phi\left[c^{-1} \operatorname{dist}\left(x_{1}, Y\right)+\varepsilon\right] \mu\left(A_{1}\right)<1\right\}\right. \\
& =\varepsilon+\operatorname{Inf}\left\{c>0: \int_{0}^{1} \phi\left[c^{-1} \operatorname{dist}\left(f^{\prime}(t), Y\right)+\varepsilon\right] d \mu(t)<1\right\} \\
& <\varepsilon+\inf \left\{c>0: \int_{0}^{1} \phi\left(c^{-1} \operatorname{dist}(f(t), Y)+\left\|f(t)-f^{\prime}(t)\right\|+\varepsilon\right) d \mu(t)<1\right\} . \\
& <\varepsilon+\inf \left[c>0: \int_{0}^{1} \phi\left(c^{-1} \operatorname{dist}(f(t), Y)+2 \varepsilon\right) \mathrm{d} \mu(t)<1\right\} \text {. }
\end{aligned}
$$

Since $\varepsilon$ is arbitrary, we have

$$
\operatorname{dist}\left(f, L_{\phi}(\mu, Y)\right)<\inf \left\{c>0: \int_{0}^{1} \phi\left(c^{-1} \operatorname{dist}(f(t), Y)\right) \operatorname{d} \mu(t)<1\right\} .
$$

REMARK 2.1. For $\mathrm{f} \varepsilon \mathrm{L}_{\phi}(\mu, \mathrm{X})$,

$$
\|f\|_{\phi}=\inf \left\{c>0: \int_{0}^{1} \phi\left(\frac{\|f(t)\|}{c}\right) d \mu(t)<1\right\}=c_{0}
$$

such that $\int_{0}^{1} \phi\left(\frac{\|f(t)\|_{0}}{C_{0}}\right) d \mu(t)=1$. 
COROLLARY 2.1. Let $Y$ be a closed subspace of $X$. To an element $f$ of $L_{\phi}(\mu, X), g$ of $L_{\phi}(\mu, Y)$ is a best approximant of $f$ in $L_{\phi}(\mu, Y)$ if and only if $g(t)$ is a best approximant of $f(t)$ in $Y$.

PROOF. Let $g(t)$ be a best appoximant of $f(t)$ in $Y$. This means that

$$
\|f(t)-g(t)\|<\|f(t)-y\| \text { for al1 } t \text { and for all y } \varepsilon \text { Y. }
$$

It follows that for any $h \in L_{\phi}(\mu, Y)$

$$
\|f(t)-g(t)\|<\|f(t)-h(t)\| \text { for a11 } t \text {. }
$$

Since $\phi$ is increasing, we have

$$
\phi\left(c^{-1}|| f(t)-g(t) \|\right)<\phi\left(c^{-1}|| f(t)-h(t) \|\right) \text { for any } c>0 \text {. }
$$

Then

$$
\int_{0}^{1} \phi\left(c^{-1}\|f(t)-g(t)\|\right) d \mu(t)<\int_{0}^{1} \phi\left(c^{-1}\|f(t)-h(t)\|\right) d \mu(t) .
$$

Therefore

$$
\begin{array}{r}
\operatorname{Inf}\left\{c>0: \int_{0}^{1} \phi\left(c^{-1}|| f(t)-g(t) \|\right) d \mu(t)<1\right\}<\inf \left\{c>0: \int_{0}^{1} \phi\left(c^{-1} \| f(t)\right.\right. \\
-h(t)|| d \mu(t) \leq 1\}
\end{array}
$$

or

$$
\|f-g\|_{\phi} \leqslant\|f-h\|_{\phi} \text { for a } 11 h \varepsilon L_{\phi}(\mu, Y)
$$

Conversely, let $g$ be a best approximant of $f$ in $L_{\phi}(\mu, Y)$, then $\operatorname{dist}\left(f, L_{\phi}(\mu, Y)\right)=\|f-g\|_{\phi} \quad$ By Lemma 2.2 and the previous remark, we have $\|\mathrm{f}-\mathrm{g}\|_{\phi}=\inf \left\{\mathrm{c}>0: \int_{0}^{1} \phi\left(c^{-1} \operatorname{dist}(\mathrm{f}(t), \mathrm{Y})\right) \mathrm{d} \mu(t)<1\right\}=c_{0}$ such that $\int_{0}^{1} \phi\left(\frac{|f(t)-g(t)| \mid}{c_{0}}\right) d \mu(t)=\int_{0}^{1} \phi\left(\frac{(d 1 s t(f(t), Y)}{c_{0}}\right) d \mu(t)=1$. Hence $\int_{0}^{1}\left[\phi\left(c_{0}^{-1}\|f(t)-g(t)\|\right)-\phi\left(c_{0}^{-1} \operatorname{dist}(f(t), Y)\right)\right] d \mu(t)=0$ since $\phi$ is strictly increasing and $\phi\left(c_{0}^{-1}|| f(t)-g(t) \|\right)>\phi\left(c_{0}^{-1} \operatorname{dist}(f(t), Y)\right)$ then ||$f(t)-g(t)||=\operatorname{dist}(f(t), Y)$.

Now we prove the main theorem of this paper.

THEOREM 2.1. Let $\mathrm{Y}$ be a closed subspace of $\mathrm{X}$, then the following are equivalent: 
(i) $\mathrm{L}_{\phi}(\mu, \mathrm{Y})$ is proximinal in $\mathrm{L}_{\phi}(\mu, \mathrm{X})$

(ii) $L^{1}(\mu, Y)$ is proximinal in $L^{1}(\mu, X)$.

PROOF. (ii) $+(\mathrm{i})$. Let $\mathrm{f} \in \mathrm{L}_{\phi}(\mu, \mathrm{X})$, then by Lemma $2.1 \mathrm{f} \in \mathrm{L}^{1}(\mu, \mathrm{X})$. By the assumption, there exists $g \in L^{1}(\mu, Y)$ such that

$$
\|\mathrm{f}-\mathrm{g}\|_{1}<\|\mathrm{f}-\mathrm{h}\|_{1} \text { for every } \mathrm{h} \in \mathrm{L}^{1}(\mu, \mathrm{Y}) \text {. }
$$

By lemma 2.10 [3], we have

$$
\|f(t)-g(t)\|<\|f(t)-y\| \text { for all } t \text { and for all } y \varepsilon Y \text {. }
$$

Hence by Corollary 2.1 it follows that $g$ is a best approximant of $f$ in $L_{\phi}(\mu, Y)$.

Conversely: $(i) \rightarrow(i i)$. Define a map

$$
J: L^{1}(\mu, X) \rightarrow L_{\emptyset}(\mu, X) \text { by } J(f)=\hat{f} \text { where } \hat{f}(t)=\frac{\emptyset^{-1}(\|f(t)\|)}{\|f(t)\|} f(t)
$$

if $f(t) \neq 0$, and zero otherwise. Then for $c=1$

$$
\begin{aligned}
\int_{0}^{1} \phi\left(\left\|c^{-1} \hat{f}(t)\right\|\right) d \mu(t) & =\int_{0}^{1} \phi\left(\left\|\phi^{-1}|\|\mid f(t)\|)\right.\right. \\
& \left.=\int_{0}^{1} \phi(t)\|f(t)\|\right) d \mu(t)
\end{aligned}
$$

for all $f \in L^{1}(\mu, X)$. Hence $J(f) \in L_{\phi}(\mu, X)$. Since $\phi$ is strictly increasing, it follows that $J$ is $(1-1)$. To show that $J$ is onto, let $g \in L_{\phi}(\mu, X)$, then take

$$
f(t)=\frac{\phi(\|g(t)\|)}{\| g(t)} \| g(t)
$$

If $g(t) \neq 0$ and zero otherwise. Clear1y $f \in L^{1}(\mu, X)$ and

$$
\begin{aligned}
J(f) & =\frac{\phi^{-1}\|f(t)\|^{1}}{\| f(t)} f(t) \\
& =\frac{\phi^{-1}(\phi(\|g(t)\|)}{\phi(\|(t)\|)} \quad \phi\left(\left\|\frac{g(t)}{}\right\|\right) \\
& =g(t) .
\end{aligned}
$$

Thus $J$ is onto. Now let $f \in L^{1}(\mu, X)$, then $\hat{f} \in L_{\phi}(\mu, X)$. By assumption there exists $\hat{g} \in L_{\phi}(\mu, Y)$ such that 


$$
\|\hat{f}-\hat{g}\|_{\phi}<\|\hat{f}-\hat{h}\|_{\phi} \text { for all } \hat{h} \varepsilon L_{\phi}(\mu, Y) \text {, }
$$

then by Corollary 2.1 we have

$$
\begin{aligned}
& \|\hat{f}(t)-\hat{g}(t)\|<\|\hat{f}(t)-y\| \text { for a1l y } \varepsilon Y \text { or } \\
& \left\|f(t)-\frac{\|f(t)\| \phi^{-1}(\|g(t)\|)}{\|g(t)\| \phi^{-1}(\|f(t)\|)} g(t)\right\|<\left\|f(t)-\frac{y\|f(t)\|}{\phi^{-1}(\|f(t)\|}\right\| \text { for }
\end{aligned}
$$

all y $\varepsilon$ Y. Pur $w(t)=\frac{\|f(t)\| \phi^{-1}(\|g(t)\|}{\|g(t)\| \phi^{-1}(\|f(t)\|)} g(t)$.

Using the facts that $\|g(t)\|<2\|f(t)\|$ since $0 \varepsilon Y$ and $\phi^{-1}(2\|f(t)\|)<2\left(\phi^{-1}(\|f(t)\|)\right)$ we can show that $w \varepsilon L^{1}(\mu, Y)$ as follows

$$
\begin{aligned}
\|w(t)\| & =\frac{\|f(t)\| \phi^{-1}(\|g(t)\|)}{\phi^{-1}(\|f(t)\|)} \\
& <\frac{\|f(t)\| \phi^{-1}(2\|f(t)\|)}{\phi^{-1}(\|f(t)\|)} \\
& <\frac{2\|f(t)\| \phi^{-1}(\|f(t)\|)}{\phi^{-1}(\|f(t)\|)} \\
& =2\|f(t)\| .
\end{aligned}
$$

Now take any $h \in L^{1}(\mu, Y)$ then

$$
\Phi^{-1}\left(\left\|\frac{f(t)}{f(t)}\right\|^{1)} h(t) \in Y \quad \text { for all } t\right. \text {. }
$$

Hence

$$
\begin{aligned}
& \|f(t)-w(t)\|<\| f(t)-\frac{\|f(t)\|}{\phi^{-1}(\|f(t)\|)} \text { 类 }\|f(t)\|^{2} h(t) \| \\
& =\|f(t)-h(t)\| \text { for all } t \text { and for all } h \varepsilon L^{1}(\mu, Y) \text {, so } L^{1}(\mu, Y) \text { is }
\end{aligned}
$$
proximinal in $L^{1}(\mu, X)$.

As a corollary.

COROLLARY 2.2. If $Y$ is a reflexive subspace of $X$, then $L_{\phi}(\mu, Y)$ is priximinal in $\mathrm{L}_{\phi}(\mu, \mathrm{X})$.

PROOF. It follows from the main theorem and Theorem 1.2 in Kahal11 [5].

THEOREM 2.2. Let $Y$ be a proximinal subspace of $X$. Then for every simple function $f \varepsilon L_{\phi}(\mu, X), \quad P\left(f, L_{\phi}(\mu, Y)\right)$ is not empty.

PROOF. Let $\mathrm{F}=\sum_{1=1}^{n} x_{A_{1}} x_{1}$ be a simple function in $L_{\phi}(\mu, x)$, where $A_{i}$ are disjoint measurable sets in $[0,1]$. Set $g=\sum_{1=1}^{n} x_{1} y_{1}$, where $y_{1} \varepsilon P\left(x_{1}, Y\right)$. Let $h$ be any 
element in $\mathrm{L}_{\phi}(\mu, \mathrm{Y})$, then

$$
\begin{aligned}
& \|f-h\|_{\phi}=\inf \left\{c>0: \int_{0}^{1} \phi\left(\left\|c^{-1}(f(t)-h(t))\right\|\right) d_{\mu}(t)<1\right\} \\
& =\inf \left\{c>0: \sum_{i=1}^{n} \int_{A_{1}} \phi\left(\left\|c^{-1}(f(t)-h(t))\right\|\right) d \mu(t)<1\right\} \\
& =\operatorname{lnf}\left\{c>0: \sum_{1=1}^{n} \int_{A_{1}} \phi\left(\left\|c^{-1}\left(x_{1}-h(t)\right)\right\|\right) d_{\mu}(t)<1\right\} \\
& >\operatorname{lnf}\left\{c>0: \sum_{1=1}^{n} \int_{A_{1}} \phi\left(\left\|c^{-1}\left(x_{1}-y_{1}\right)\right\|\right) d \mu(t)<1\right\} \\
& =\operatorname{lnf}\left\{c>0: \int_{0}^{1} \phi\left(\left\|c^{-1}(f(t)-g(t))\right\|\right) d \mu(t)<1\right\} \\
& =\|f-g\|_{\phi} .
\end{aligned}
$$

Hence $\mathrm{g} \varepsilon \mathrm{P}\left(\mathrm{f}, \mathrm{L}_{\phi}(\mu, \mathrm{Y})\right)$.

THEOREM 2.3. Let $Y$ be a closed subspace of $X$. If $L_{\phi}(\mu, Y)$ is proximinal in $L_{\phi}(\mu, X)$, then $Y$ is proximinal in $X$.

PROOF. From Theorem 2.1, $L_{\phi}(\mu, Y)$ proximal in $L_{\phi}(\mu, X)$ implies that $L^{1}(\mu, Y)$ is proximinal in $L^{1}(\mu, X)$. By Theorem 1.1 [2] this also implies that $L^{\infty}(\mu, Y)$ is proximinal in $\mathrm{L}^{\infty}(\mu, \mathrm{X})$. For $\mathrm{x} \varepsilon \mathrm{X}$, define $\mathrm{f}_{\mathrm{x}}: \Omega+\mathrm{X}$ by $\mathrm{f}_{\mathrm{x}}(t)=\mathrm{x}$ for all $t \varepsilon \Omega$. It is clear that $f_{x} \in L^{\infty}(\mu, X)$ for every $x \in X$, so there exists $h \varepsilon L^{\infty}(\mu, Y)$ such that

$$
\left\|f_{x}-h\right\|_{\infty}<\left\|f_{x}-w\right\| \text { for every } w \varepsilon L^{\infty}(\mu, Y)
$$

In particular take $w=f_{y}$, so

$$
\begin{aligned}
\left\|f_{x}-h\right\|_{\infty} & <\left\|f_{x}-f_{y}\right\|_{\infty} \text { for every y } \varepsilon Y \\
& =\|x-y\| \quad \text { for every y } \varepsilon Y .
\end{aligned}
$$

But $\|x-h(t)\|=\left\|f_{x}(t)-h(t)\right\|$

$$
\begin{gathered}
<\left\|f_{x}-h\right\| \\
<\left\|f_{x}-f_{y}\right\| \\
=\|x-y\| \quad \text { for every } y \in Y .
\end{gathered}
$$

Hence every $t \varepsilon[0,1]$ gives a best approximant of $x$ in $Y$. Therefore $Y$ is proximinal in $X$.

The next theorem needs the following definitions: 
DEFlnItION 2.1. The subspace $Y$ is called $\phi$-summand of $x$ if there is a bounded projection Q: $\mathrm{X} \rightarrow \mathrm{Y}$ such that

$\phi(\|x\|)=\phi(\|(Q(x) \|)+\phi(\|(I-Q)(x)\|)$ for all $x \in X$. Where $I$ is the identity map on $x$.

DEFINITION 2.2. The subspace $Y$ is called 1 -complemented in $X$ if there is a closed subspace $Z$ In $X$ that $X=Y+Z$ and the projection $P$ : $X+Z$ is a contractive projection.

THEOREM 2.4. If $Y$ is 1 -complemented in $X$, the $L_{\phi}(\mu, Y)$ is proximinal in $L_{\phi}(\mu, X)$.

PROOF. Let $X=Y \pm Z, P: X \rightarrow Z$ be a contractive projection from $X$ onto $Z$. Hence $x=(L-P) x+p(x), \quad\|P(x)\|<\|x\|$. For $f \in L_{\phi}(\mu, X)$, set $f_{:}=(I-P) \cap f, f_{i}:$ pof. Let $\breve{p}: L_{\phi}(\mu, X)+L_{\phi}(\mu, Z)$ and

$$
p(f)=p o f=f_{2} \text { for all } f \varepsilon L_{\emptyset}(\mu, X) .
$$

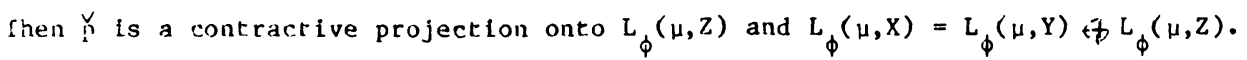
Hence $L_{\phi}(\mu, Y)$ is 1 -complemented in $L_{\phi}(\mu, X)$. By Lemma $1.6[2] L_{\phi}(\mu, Y)$ is proximinal in $L_{\phi}(\mu, X)$.

\section{REFERENCES}

1. DEEB, W. and KHALIL, R. Best Approximation in $\mathrm{L}^{\mathrm{P}}(\mu, \mathrm{X}), 0<\mathrm{p}<1$. To appear in Journal of Approximation Theory.

2. DEEB, W. and KAHLIL, R. Best Approximation in $L(X, Y)$. To appear in Math. Proc. Camb. Phi1. Soc.

3. KHALIL, R. and DEEB, W. Best Approximation in $\mathrm{L}^{\mathrm{P}}(\mu, \mathrm{X})$ II. To appear in Journal of Approximation Theory.

4. LIGHT, W.A. and CHENEY, E.W. Approximation Theory in Tensor Product Spaces. Lecture Notes in Math. $1169,1985$.

5. KHALIL, R. Best Approximation in $\mathrm{L}^{\mathrm{P}}(\mathrm{I}, \mathrm{X})$. Math. Proc. Camb. Phil. Soc. 49 (1983), 277-279.

6. ROYDEN, H.L. Real Analysis. Macmillan Publishing Co., Inc., New York, 1968.

7. SINGER, I. Best Approximation in Normed Linear Spaces by Elements of Linear Subspaces, Springer-Verlag, 1970. 


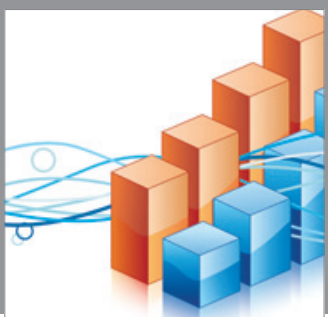

Advances in

Operations Research

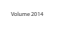

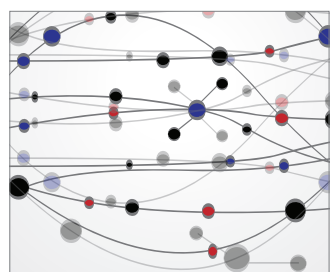

\section{The Scientific} World Journal
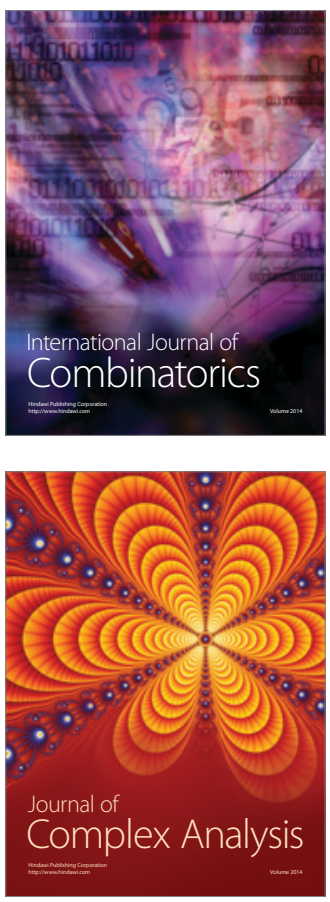

International Journal of

Mathematics and

Mathematical

Sciences
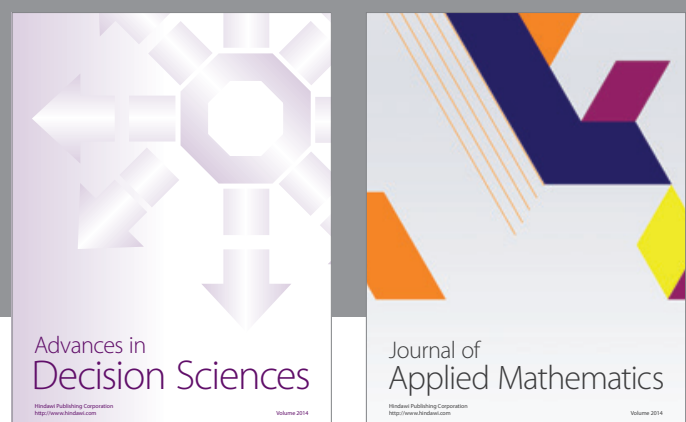

Journal of

Applied Mathematics
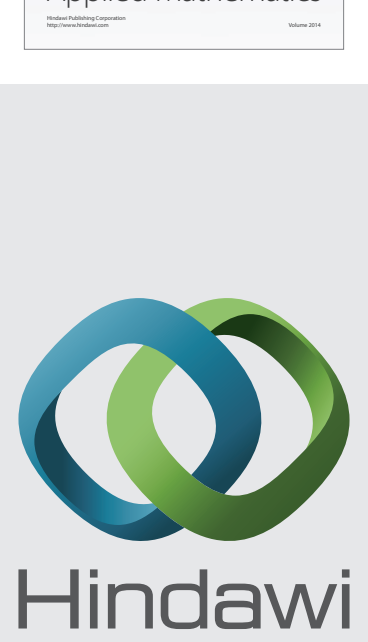

Submit your manuscripts at http://www.hindawi.com
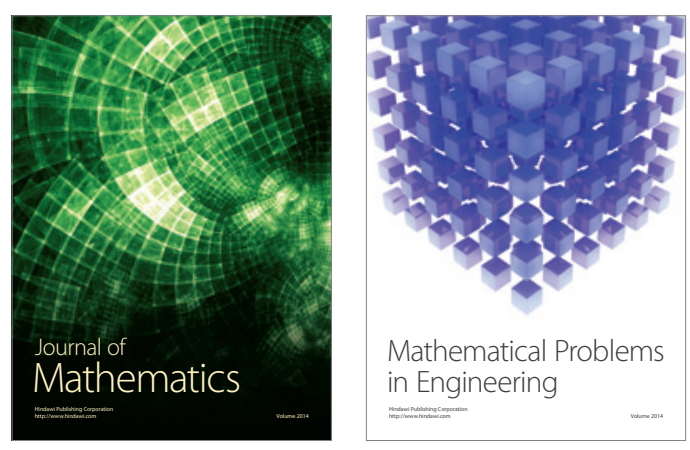

Mathematical Problems in Engineering
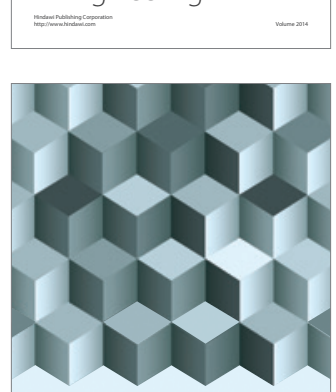

Journal of

Function Spaces
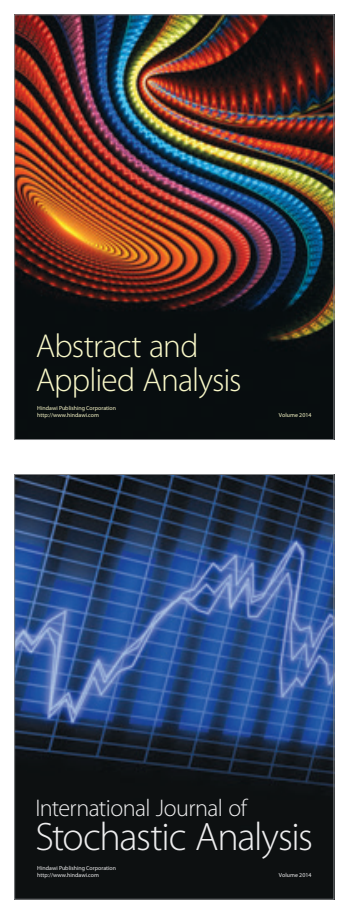

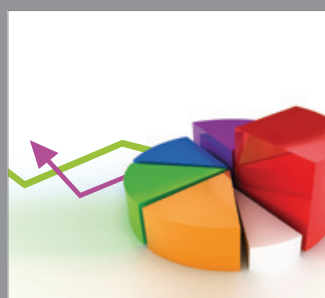

ournal of

Probability and Statistics

Promensencen
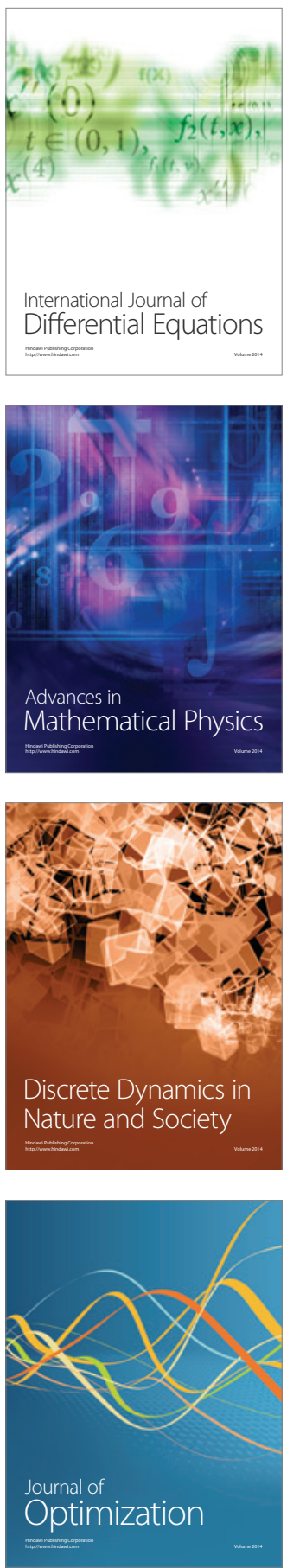